\title{
An Anchor based Data Collection Algorithm for Improving LifeTime Ratio of WSN
}

\author{
Geethanjali R. C. \\ Digital Electronics \& Communication Systems \\ VIAT, Chickbalapur, India
}

\author{
Sarika Tale, $\mathrm{PhD}$ \\ Digital Electronics \& Communication Systems \\ VIAT, Chickbalapur, India
}

\begin{abstract}
Wireless device networks have vividly been used in various different applications in contemporary days. The main problem with the WSN is duration of the shells/ batteries applicable to many situations. In order to overcome from this issue, very important aspect is to increase the duration of the battery therefore an additional energy stream is required. Energy from the surrounding is one of the God gift. The power from the surroundings makes the device points to backup power on its own, which approximately gives infinite duration. So in this paper we make use of a mobile collector nothing but a Sencar. It put the energy from all the points in the network. On surveying with randomized routing protocol it had lot of energy wastage. More or less to come out of the problem they are introducing broadcasting routing protocol. This protocol including the sencar and anchor based routing. At last the comparing of both the protocols ie the randomized and the broadcasting routing protocols with certain parameters like Alive points, dead points, throughput energy etc. On Comparision broadcasting routing is best when compared to the other.
\end{abstract}

\section{General Terms}

Randomized routing protocol, Broadcast routing protocol, Sencar and anchor protocol.

\section{Keywords}

Energy Harvesting, Wireless sensor network, Data gathering, Residual Energy, Ad-hoc networks.

\section{INTRODUCTION}

By way of skill developments in the social order the requirement aimed at on-line and off-line interacting which turn out to be important. Every other forms of schmoozing have its benefits as well as drawbacks in keeping with safety. On-line interacting abstains totally dissimilar hardware necessities; also vary in the advantages square measure difference. Off-line making contacts proceeds into different, mobility, and in also many forms of hardware elements that are required for determining the off-line web. Scan on perceive different kinds of conformations in set-ups, also in safety processes which requires to confirm a protected net. Administrations trusts and depends more in power in order to spread the data all over the institute in assistant cost-effective along with prolific mode. Workstation links allows in the knowledge as well as which are virtually working in the present occupation. Company allows dual options when these take part in the connecting of link. Renewable energy sources like star, wind, thermal necessitates Cyber Physical Systems (a network consists of sensors and actuators to act with the physical world) for achieving energy potency and price effectiveness. For instance, star harvest is well-tried to be helpful to supply energy to sensors from a solar battery of comparatively similar size of sensors [1]. Thermo electrical conversion offers opportunities to reap energy via heat transfer once the temperatures of objects or environments area unit totally different [2]. Once power from associate degree close energy supply is brought into a WSN, it becomes reversible. An ad-hoc or peer-to-peer off-line connection has many different types of mainframes every 1 equipped along the off-line connection boundary postcard. Each laptop easily interconnects straight with the off-line permitted PC's placed in front. They'll have the capability to share files and printers, however it might be impossible to use the on-lined computer network assets, without or within the mainframes can act like a path way for another on-line computer network victimization package of software.

Objective of the paper is to design an optimization agenda such that data gathering of network utility is increased and packet latency can be bounded by an already determined threshold. To overcome this problem, they have proposed a two-step approach. First step, they found out where the Sencar stops to collect the data packets while confirming that the total migration tour length is bounded by the threshold. Then these node positions are called anchors. Second step, once the anchors has been selected, then they calculated the optimization problem into a network utility maximization problem on the bases of the constraints of flow, energy balance, battery and alive nodes.

\section{RELATED WORK}

Improvements in harvesting energy devices have provided many different methods to extend network duration of WSNs by making use of renewable energy from environment. Ecofriendly vitality harvest home, specifically star based mostly, had begun with variety of methods to find alternative shell provides. Moreover, coming up with an economical star harvest home pc to appreciate the probable advantages of energy harvest home needs an very high thoughtful of many factors. Putting forward the mobility in energy harvesting WSNs was considered in [1]. In the system, a tiny low proportion of net points square measure originally mobile, permitting everybody to maneuver in exploration of energy, boost, and send energy to permanent, energy depleted points. They need termed this approach energy gathering. They need characterized the matter of bumpy energy feeding, counsel energy gathering as potential answer, and supply an easy analytical framework to judge energy consumption theme. Information from initial practicableness experiments exploitation energy gathering show promising results.

Maria Gorlatova, Aya Wallwater has told in a Paper, in this they need delineate and explained the changes in energy gather ingredients and ultralow power transportations can presently modify the belief of nets self-possessed of energy gather strategies. Such devices can run victimization terribly less close energy, like interior light-weight drive. They centered on exemplifying of energy convenience in interior 
atmospheres and in unindustrialized energy sharing procedures for energy gather devices [4]. First, the given results of their long indoor energy measurements, which give vital $\mathrm{i} / \mathrm{ps}$ needed for algorithmic rule \& pc style (ex., determinant the specified battery masses). Then, they centered on algorithmic rule called enlargement, that needs nontraditional lines in energy gather swings the character of energy aware rules from decreasing energy outlay in maintaining it. However, in several ways, totally diverse energy stowage varieties need diverse systems. They need developed algorithms for determinant time truthful energy provision in the $\mathrm{PC}$ for expecting inputs.

Christopher M. Vigorito, Deepak Ganesan have given elaborate plan concerning the present techniques to handle this downside square measure minimally adaptive and use a lot of priori information of the energy shape. Whereas methods square measure cheap in atmospheres with less adjustment, they discovered it's extremely wasteful in additional capricious eventualities. They tend to make known to a replacement procedure in resolving the downside supported grades for adaptive management system and they tend to reach higher concert when compared to before methods on outline category of energy supply knowledge sets [9]. In addition, they tend to embody tumble machinery in order to reduce the changes in the points in duty change over duration that is a very generic article in responsibilities like event observation. They tend to get reduce in modification as nice as common fraction while not bargaining duty recital to take care of energy impartial act.

\section{METHODOLOGY}

In order to implement this project many algorithms were proposed like Randomized Algorithm, Broadcasted Randomized Protocol, Sencar and Anchor Based Routing, Anchor Selection Algorithm. The Randomized Algorithm is the old method which was proposed for energy harvesting in wireless sensor networks. The shown figure1 gives the system architecture of energy harvesting WSN. The Node Deployment is the algorithm which is used to place the Nodes in the 8 different regions and each of the regions consist of at least 5 Nodes. Formation of routing table is used to form the routing tables for the various nodes in the network. Network Neighbor Nodes is used to determine the IN Nodes in the Network. These are the set of Nodes which are in the GPS Range of other Nodes in the network. Randomized Protocol is the path determination algorithm which is used to form the path from sender Node to destination Node securely and undeterministic manner.

Broadcasted randomized protocol is used to reduce the consumption of energy in the WSN. Many other distributed algorithms are discussed in various papers, in order to overcome the disadvantages in randomized protocols, the broadcasted randomized protocol came into picture i e Figure 5. 1) Initial Node, List of end Nodes and Communication Range as input.2) Start from first destination node 3) Check whether current destination node is last in list. 4) Pick the current destination node5) Perform randomized route discovery in the middle of the start node and current last node6) Store the route in the cache, 7) Repeat from Step 2 to Step6 until all routes are found out.

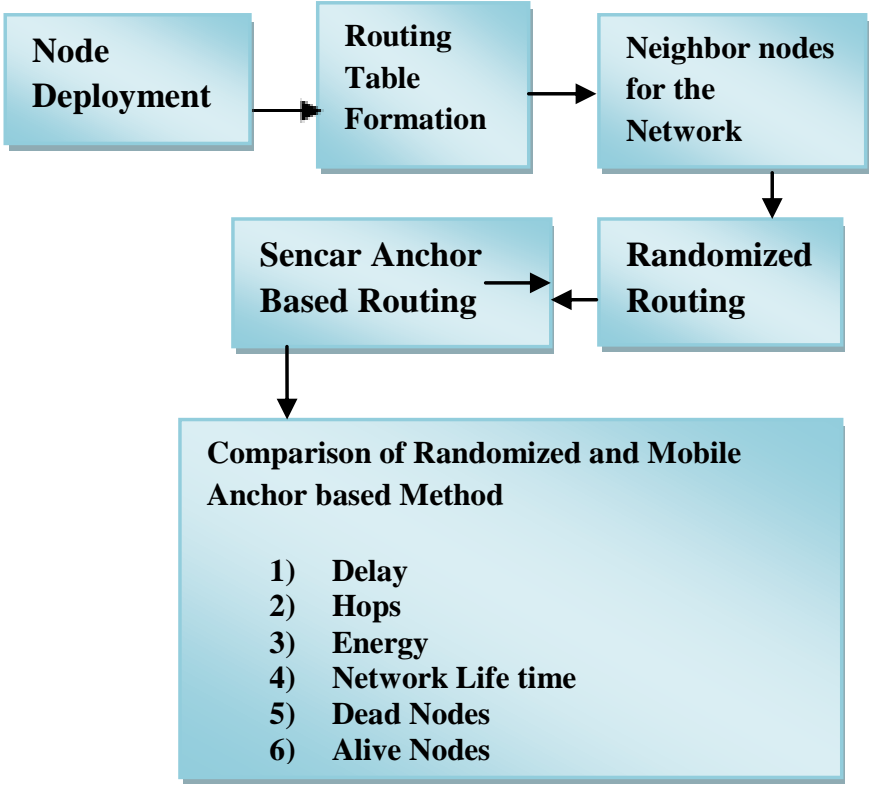

Fig 1: System Architecture of Energy Harvesting Wireless Sensor Network

From Figure 6 shows the routing of Sencar and Anchor nodes 1) The Source Node, Sencar Node and Anchor List 2) Find the number of count of Anchor List 3) Communicate from the sencar node to the anchor node 4) Repeat the process for all the anchor nodes.

\subsection{Comparision Parameters}

Destination to destination Delay is the time taken for the RREQ to go from the source node to destination node and then send back the RRPLY from destination node to source node.

$$
E 2 E_{\text {delay }}=t_{\text {stop }}-t_{\text {start }}
$$

Where,

$t_{\text {stop }}=$ This is the Time at which RRPLY is recieved

$t_{\text {start }}=$ This is the Time at which RREQ is send

The total energy consumption is given as follows:

$$
\begin{aligned}
& T E=\sum_{i=1}^{l} E_{c}(i) \\
& \text { Where, } \\
& l=\text { Number of Links } \\
& E_{c}(i)=\text { Energy consumed across } i^{\text {th }} \text { link }
\end{aligned}
$$

Number of alive nodes is given as It is defined as number of node sets where their energy level is $>I=$ to $\mathrm{B} / 4$, so that $\mathrm{B}$ may be the starting energy. Dead nodes are defined as number of node sets where battery level is less than $B / 4$ Where $B$ is initial Battery Power.

Routing Overhead $=\frac{\text { Num of control packets }}{\text { Num of Data packets }}$ 


\section{RESULTS AND ANALYSIS}

There square measure some fascinating problems to be explored more in future. First, a way to apply the planned framework to a network of enormous size wherever multiple sencars square measure required to make sure ascendable knowledge assortment. During this case, partitioning of the network into completely different regions such every Sencar solely deals with a little of the network takes place. Second, the improvement framework might be additional improved. The anchor choice rule will take a look at additional combinations of anchors at intervals the tour length sure. This may permit the Sencar to gather additional information and enhance network utility. Moreover, joint improvement that considers all choices of anchors and selects the most effective network utility is additional fascinating. Additionally, the improvements algorithms are often additional improved to account for future energy financial gain and expenditures therefore the harvested energy are often properly utilized within the current measure.

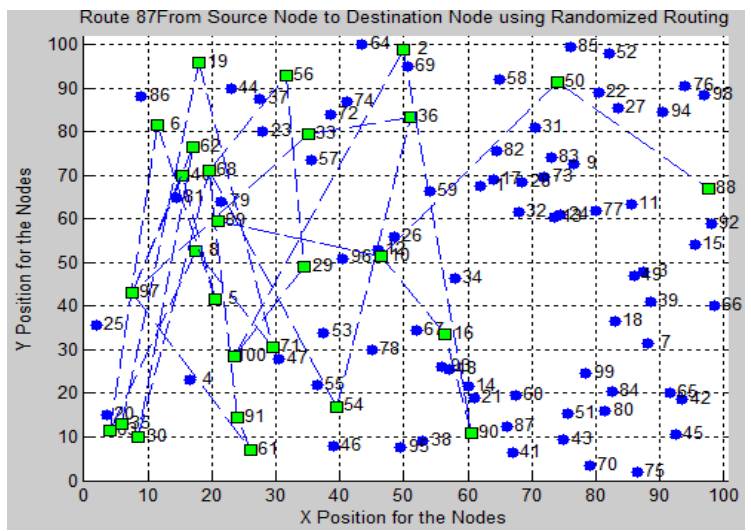

Figure 2: Source node to Destination node using Randomized Routing

From the figure 2 it shows the transfer of packets from the source nodes to the destination node using Randomized routing protocol. Lot of energy is wasted when the data is transferred. But were as in figure 3 the complete area is divided into four regions in which anchor is selected from each region so that energy is not reduced. Figure 4(a) shows the performance alive nodes in which the number of alive nodes is more in anchor based routing when compared to randomized routing protocol, From figure 4(b) and 4(c) clearly shows that the anchor based routing protocol is best compared to randomized routing protocol in terms of performance throughput and Residual energy.

\section{CONCLUSION AND FUTURE WORK}

In this paper the 2 algorithms namely Randomized Routing and Sencar based routing algorithms have been described. Randomized Routing algorithm is used to find neighbors and then pick who reply time is lowest acting like the receiver. Sencar based routing algorithm performs the data collection by using a weight factor computation for selection of anchor nodes and then the data is collected by using combination of anchor nodes and mobile sink.

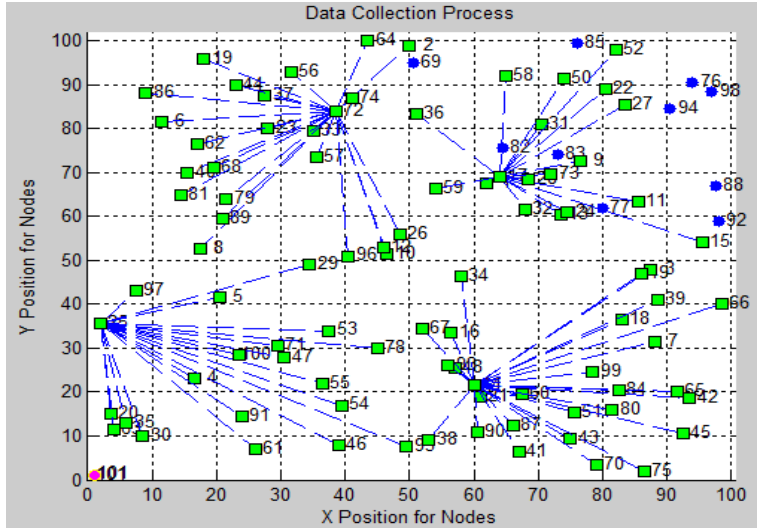

Figure 3: Data Collection process in Anchor Based Protocol

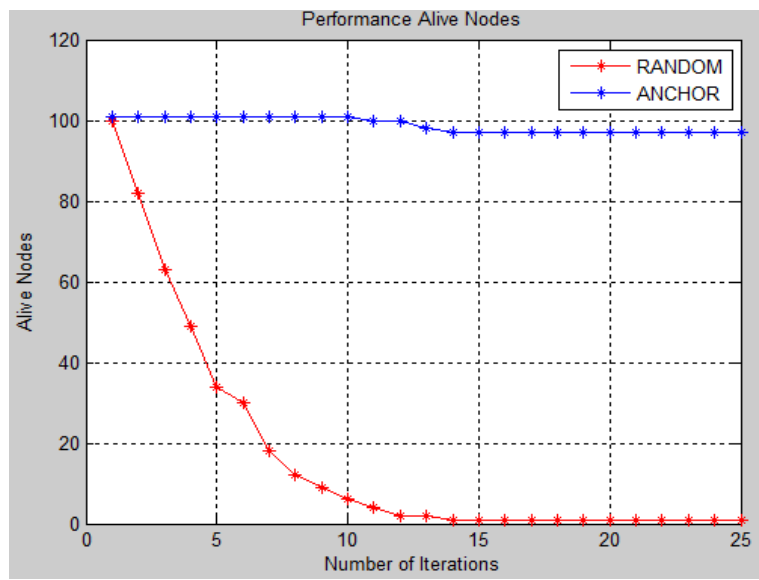

Figure 4(a)

Finally comparison is performed between randomized routing and Sencar based routing algorithm and proved that proposed method is the best with respect to the following parameters namely End to End Delay, Number of Hops. Energy Consumed, Number of Alive Node, Number of Dead Nodes, Residual Energy.Lifetime Ratio and Routing Overhead like this process is repeated until the destination is reached. Like this process is repeated with each node.

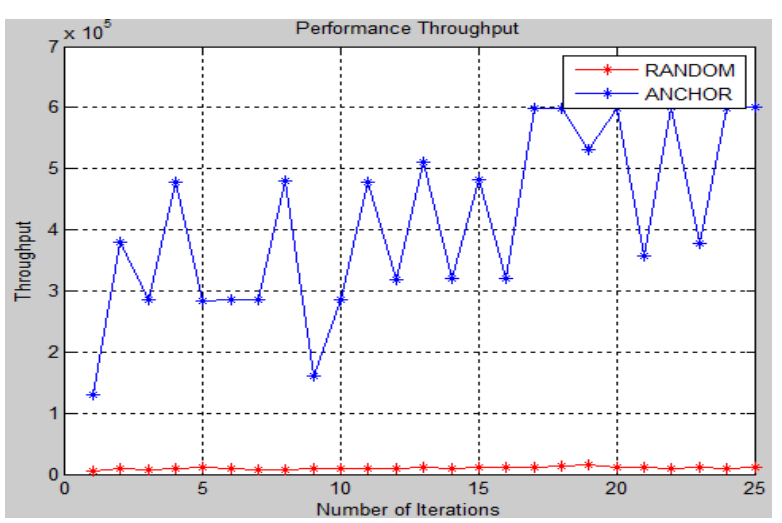

Figure 4(b) 


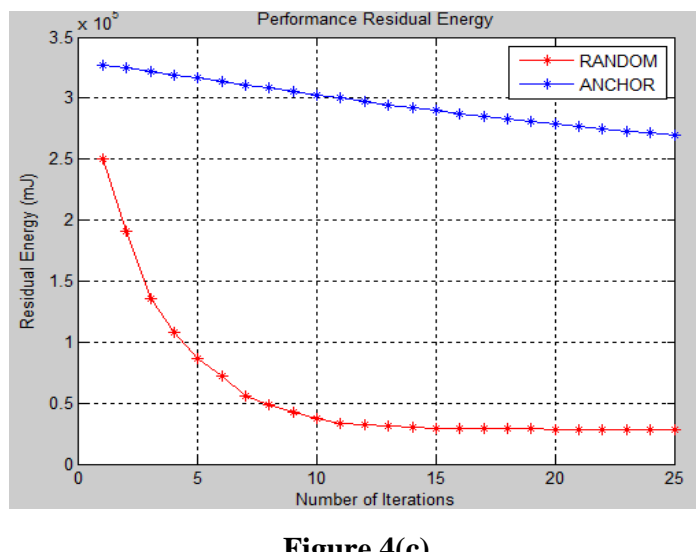

Figure 4(c)

Fig 4: (a) Performance Alive Nodes (b) Performance Throughput (c) Performance Residual Energy

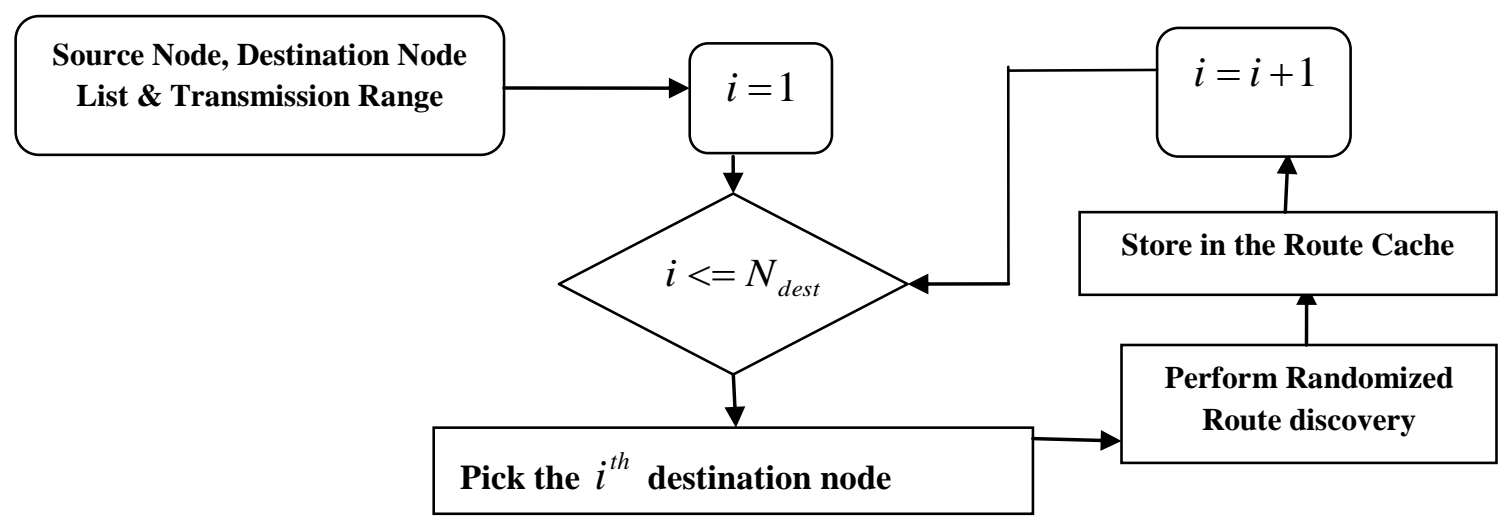

Figure 5: Broadcast Route Discovery Randomized Algorithm

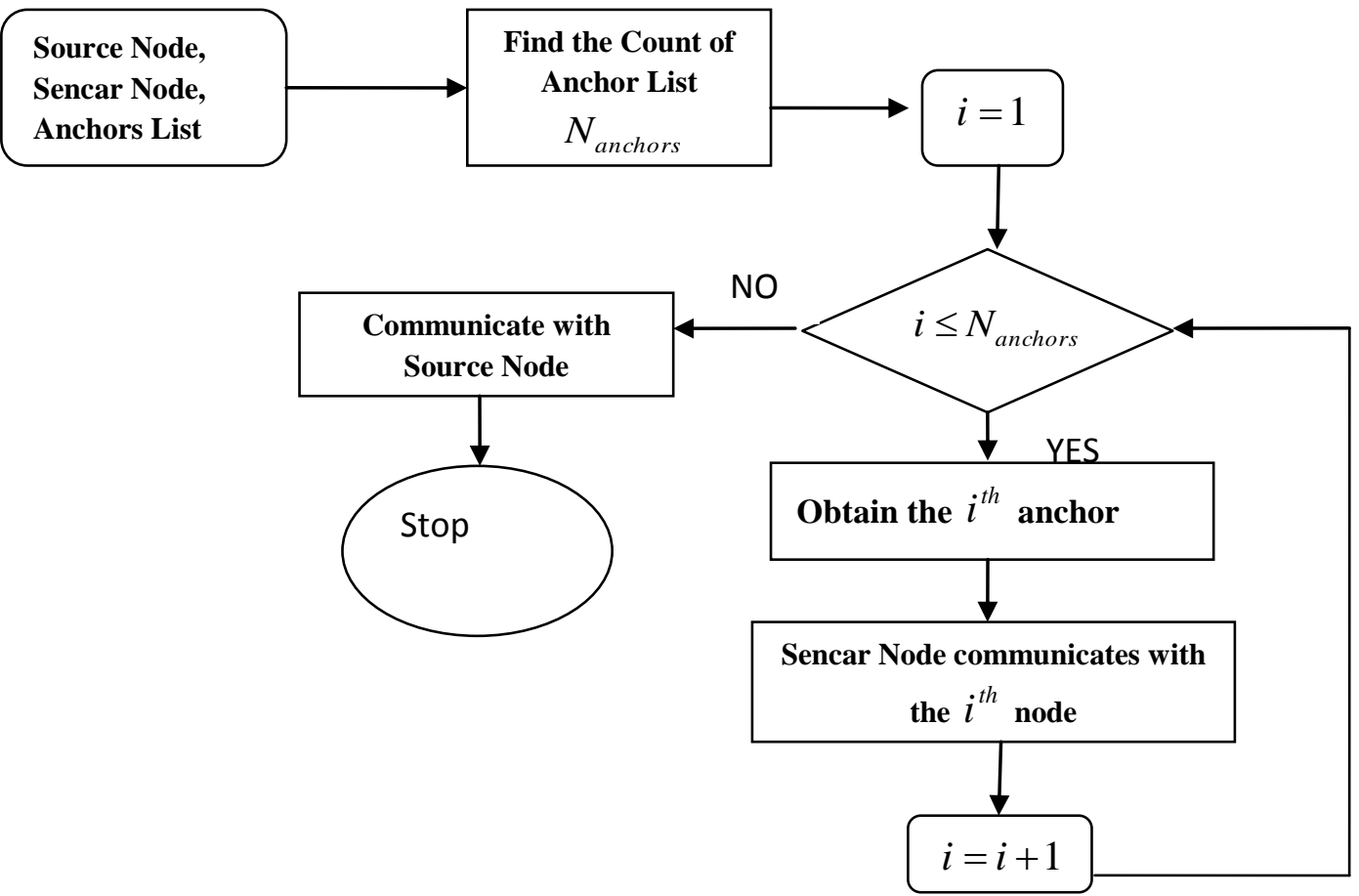

Figure 6: Flowchart of Sencar and Anchor based Routing 


\section{REFERENCES}

[1] M. Rahimi, H. Shah, G. Sukhatme, J. Heideman, and D. Estrin, "Studying the feasibility of energy harvesting in a mobile sensor network," in Proc. IEEE Int. Conf. Robot. Autom., 2003, pp. 19-24.

[2] J. Paradiso and T. Starner, "Energy scanverging for mobile and wireless electronics," IEEE J. Pervasive Comput., vol. 4, no. 1, pp. 18-27, Jan. 2005.

[3] X. Jiang, J. Polastre, and D. Culler, "Perpetual environmentally powered sensor networks," in Proc. 4th Int. Symp. Inf. Process.Sens. Netw. Apr. 2005, pp. 463468.

[4] V. Raghunathan, A. Kansal, J. Hsu, J. Friedman, and M. Srivastava, "Design considerations for solar energy harvesting wireless embedded systems," in Proc. 4th Int. Symp. Inf. Process. Sens. Netw., Apr. 2005, pp. 457462.
[5] C. Ma, Z. Zhang, and Y. Yang, "Battery-aware scheduling in wireless mesh networks," Mobile Netw. Appl., vol. 13, no. 1-2, pp. 228-241, 2008.

[6] C. Ma and Y. Yang, "Battery-aware routing for streaming data transmissions in wireless sensor networks," Mobile Netw. Appl., vol. 11, no. 5, pp. $757-$ 767, 2006.

[7] A. Kansal, J. Hsu, M. Srivastava, and V. Raqhunathan, "Harvesting aware power management for sensor networks," in Proc. 43rd ACM/IEEE Des. Autom. Conf., 2006, pp. 651-656.

[8] C. Ma and Y. Yang, "A battery-aware scheme for routing in wireless ad hoc networks," IEEE Trans. Veh. Technol., vol. 60, no. 8,pp. 3919-3932, Oct. 2011.

[9] C. Vigorito, D. Ganesan, and A. Barto, "Adaptove control of duty cycling in energy-harvesting wireless sensor networks," in Proc. 4th Annu. IEEE Commun. Soc. Conf. Sensor, Mesh Ad Hoc Commun.Netw., 2007, pp. 21-30. 